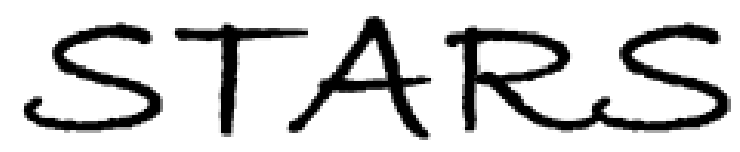

University of Central Florida

STARS

$1-1-2009$

\title{
Droplet formation and stability of flows in a microfluidic T-junction
}

Amit Gupta

University of Central Florida

S. M. Sohel Murshed

University of Central Florida

Ranganathan Kumar

University of Central Florida

Find similar works at: https://stars.library.ucf.edu/facultybib2000

University of Central Florida Libraries http://library.ucf.edu

This Article is brought to you for free and open access by the Faculty Bibliography at STARS. It has been accepted for inclusion in Faculty Bibliography 2000 s by an authorized administrator of STARS. For more information, please contact STARS@ucf.edu.

\section{Recommended Citation}

Gupta, Amit; Murshed, S. M. Sohel; and Kumar, Ranganathan, "Droplet formation and stability of flows in a microfluidic T-junction" (2009). Faculty Bibliography 2000s. 1602.

https://stars.library.ucf.edu/facultybib2000/1602

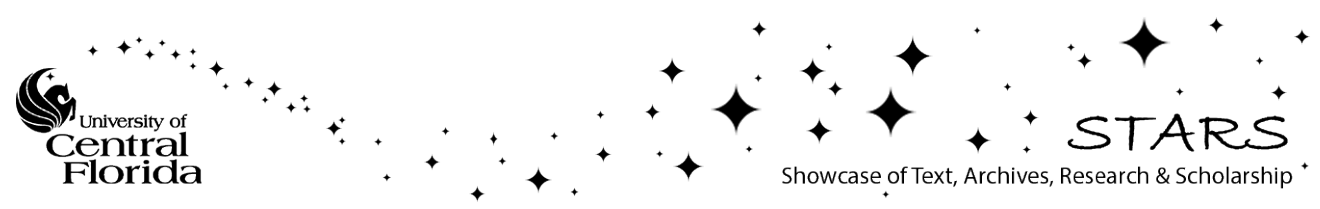




\section{Droplet formation and stability of flows in a microfluidic T-junction}

Cite as: Appl. Phys. Lett. 94, 164107 (2009); https://doi.org/10.1063/1.3116089

Submitted: 03 March 2009 . Accepted: 14 March 2009 . Published Online: 23 April 2009

Amit Gupta, S. M. Sohel Murshed, and Ranganathan Kumar

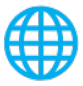

\section{ARTICLES YOU MAY BE INTERESTED IN}

\section{Droplet formation in a T-shaped microfluidic junction}

Journal of Applied Physics 106, 034906 (2009); https://doi.org/10.1063/1.3187831

Formation of dispersions using "flow focusing" in microchannels

Applied Physics Letters 82, 364 (2003); https://doi.org/10.1063/1.1537519

Droplet formation in microfluidic cross-junctions

Physics of Fluids 23, 082101 (2011); https://doi.org/10.1063/1.3615643

\section{Applied Physics Letters}

Mid-IR and THz frequency combs special collection 


\title{
Droplet formation and stability of flows in a microfluidic T-junction
}

\author{
Amit Gupta, S. M. Sohel Murshed, and Ranganathan Kumar ${ }^{a)}$ \\ Department of Mechanical, Materials and Aerospace Engineering, University of Central Florida, Orlando, \\ Florida 32816, USA
}

(Received 3 March 2009; accepted 14 March 2009; published online 23 April 2009)

\begin{abstract}
Flow regimes obtained as a consequence of two immiscible fluids interacting at a T-junction are presented for high Capillary numbers and different flow rates of the continuous and dispersed phases. Through lattice Boltzmann based simulations, a regime map is created that distinguishes parallel flows from droplet flows. Simulations show the dependence of flow rates and viscosity ratio on the volume of droplets formed, which is compared with existing experimental data. At high Capillary numbers, the transition zone which separates parallel and droplet flows shrinks, and is influenced by the viscosity ratio as well. () 2009 American Institute of Physics.
\end{abstract}

[DOI: 10.1063/1.3116089]

In recent years, microfluidic devices that generate emulsions of liquid droplets suspended in an outer immiscible liquid have found widespread applications in food processing, drug delivery, microanalysis, tumor destruction, as ultrasound agents, and in chemical reactions at the micron level. ${ }^{1}$ Many applications are found that involve multiphase transport through thin capillaries and microchannels. For instance, miniature evaporative and distillation units and bubble generators, flow cytometers, and emulsions all use transport of two dissimilar fluids through a microchannel. Due to the length scales involved, the flow of the dissimilar fluids falls in the laminar regime, which enables a higher degree of control on the injection process. Due to the large surface-tovolume ratio of microchannel, the flow is usually surface tension dominated. The experimental data ${ }^{2}$ show that when for a given continuous phase flow rate, if the dispersed flow is increased, the flow pattern changes from droplets at T-junction (DTJ) to droplets in channel (DC). This indicates that for a given Capillary number $\left(\mathrm{Ca}=\mu_{c} U_{c} / \sigma\right)$, when the flow rate ratio $Q\left(=Q_{d} / Q_{c}\right)$ is increased, a regime change happens. With further increase in $Q$, the flow regime changes to parallel flow (PF). ${ }^{2}$ These regimes appear to be established mostly for low Capillary numbers. This letter will show that when the surface tension forces are comparable to viscous forces, the transition to different flow regimes occur earlier and quicker. In addition to the Capillary number, the other parameters that govern droplet generation are the flow rates, viscosity ratio, and the geometry of the microchannel. This letter will also address the effect of high viscosity of the continuous fluid (low viscosity ratio). Our aim is to establish the dependence of the shear and surface tension forces for Capillary numbers that define flows in the dripping to jetting regime through numerical simulations, and quantify the results in terms of the "stable" droplet formation regime in a T-junction microchannel.

Over the past few years, numerous experiments have highlighted the advances being made in the field of droplet generation using a variety of microfluidic devices, particularly using coflowing fluids, ${ }^{3,4}$ passing dissimilar fluids through an orifice ${ }^{5-7}$ and droplet formation at T-junctions. ${ }^{2,8-11}$ Our interest in this work was initiated by

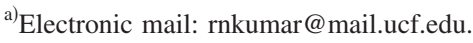

recent experimental findings which reveal that the stability of parallel flows in a funneled T-junction is linked to the flow rates, wetting behavior, viscosity contrast, and aspect ratio of the inlet channel for the two liquids. ${ }^{2}$ The geometry of the microchannel chosen was different from the conventional T-junction microchannels commonly encountered, and the droplet formation was a consequence of the competing nature of two fluid streams colliding head-on, and subsequently guided through a funnel into the main channel. ${ }^{2}$ Although theoretical work on exploring the interplay of surface tension and viscous forces for such devices has been gaining attention, ${ }^{10,12}$ these have primarily focused on viscosity ratios, $\lambda=\mu_{d} / \mu_{c}>1 / 8$, and aspect ratios $w_{c} / h=1$ and $\Lambda$ $=w_{d} / w_{c}=1$, where $w_{c}$ and $w_{d}$ are the widths of the continuous and dispersed phase channels, and $h$ is the depth. A theoretical treatment of the problem has not been carried out for $\mathrm{Ca}>0.1$, where squeezing pressure is not the dominant mechanism for droplet formation, and for low values of the viscosity ratios and dissimilar widths $w_{c}$ and $w_{d}$. Through a diffuse-interface three-dimensional lattice Boltzmann method (LBM), we present results that reveal the influence of Capillary number, viscosity ratio, and flow rate ratio on the transition from droplet flows to parallel flows in the dripping regime. To accomplish this, a recent algorithm has been implemented that models the interface dynamics of two dissimilar liquids of similar density. ${ }^{13}$ In this formulation of the stress boundary condition, the perturbation step of the original model ${ }^{14}$ is replaced by a direct forcing term at sites where the dissimilar fluids are present. A recently proposed improvement to keep numerical diffusion in check and reduce the spurious currents to $\mathrm{O}\left(10^{-5}\right)$, which is $<1 \%$ of the continuous phase velocity, is also implemented. ${ }^{15}$ The interface between the dissimilar fluids is maintained by applying a force that acts centripetally with a magnitude proportional to the gradient of the color, given by $\mathbf{F}=-\sigma / 2 c_{s}^{2} R w_{i} \mathbf{e}_{i} . \nabla C$, where $R$ is the radius of curvature of the interface, $c_{s}$ is the speed of sound in lattice units $\left(c_{s}^{2}=1 / 3\right), w_{i}$ is the weight along the link $\mathbf{e}_{i}$, and $\nabla C$ is the gradient of the color field. At the end of the LBM collision process, particle distributions for the two fluids are calculated using the antidiffusion method. ${ }^{15}$ The width of the interface is controlled using the antidiffusion parameter to remove numerical artifacts such as lattice pinning. The wetting characteristics of the main chan- 


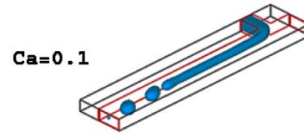

(a)

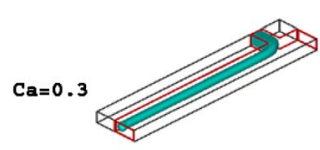

(d)

$Q=1 / 5$

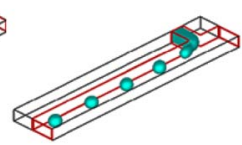

(b)

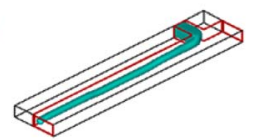

(e)

$Q=1 / 10$

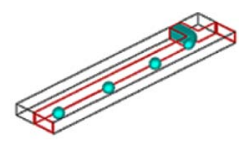

(c)

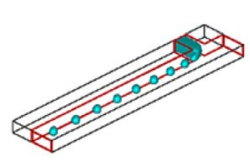

(f)

$Q=1 / 20$
FIG. 1. (Color online) Regime map of droplet formation and flow pattern in the channel for two different capillary numbers and three different flow rate ratios at fixed $\lambda=1 / 10$ : (a) jetting, (b) droplet formation with necking or thread, [(c) and (f)] droplet formation, and [(d) and (e)] parallel flow.

nel are chosen to ensure that the continuous liquid always wets the surface, whereas the dispersed phase is nonwetting on the wall.

Geometric parameters are chosen to be $\Lambda=w_{d} / w_{c}=0.5$ and $w_{c} / h=1.11$. Dynamic viscosity ratios for the continuous and dispersed phase used in this study are $\lambda=1 / 10,1 / 30$, $1 / 50$, and $1 / 100$. At each $\mathrm{Ca}$, the continuous phase flow rate was held constant while the dispersed phase flow rate was increased. For $\lambda=1 / 10$ and low value of the flow rate ratio $Q$, droplets were formed at the entrance of dispersed channel through a dripping mechanism. As $Q_{d}$ is increased, droplets were found to pinch-off downstream of the T-junction, forming a thread that becomes unstable after a distance from the dispersed channel entrance. For even higher flow rate $Q_{d}$, the protruding thread is found to be stable. The three flow patterns of DTJ, DC, and PF evolve, respectively. For a fixed $\lambda=1 / 10$, with an increase in flow rate ratio the droplet formation process [Figs. 1(c) and 1(f)] transforms to formation of a thread [Fig. 1(b)] and subsequently a stable jet [Fig. 1(a)] or parallel flow [Figs. 1(d) and 1(e)]. For the same geometry and viscosity ratio, the threshold value of flow ratio, $Q_{\text {threshold, }}$ at which a stable parallel flow appears for the first time is found to decrease as the Capillary number increases. The presence of the parallel flow is marked by shedding of a few droplets $(<5)$ before the jet fills the entire length of the main channel.

The interplay of flow rate ratio and viscosity ratio is important in determining whether the flow regime is continuous or droplet-laden. When the continuous flow viscosity is increased, i.e., for a lower $\lambda=1 / 30$, a slightly different trend emerges. For example, at $\mathrm{Ca}=0.5$, the threshold value of flow rate ratio at which a jet becomes unstable is $1 / 10$. For $\lambda=1 / 10$ for the same Capillary number, the threshold occurs at a lower $Q_{d}$, i.e., $Q_{\text {threshold }}=1 / 20$. The transition from DC to DTJ is also influenced by the viscosities of the two fluids. For $\lambda=1 / 30$, this transition to dripping occurs at $Q=1 / 12$, whereas for $\lambda=1 / 10$, the corresponding flow rate ratio is $Q$ $=1 / 24$. Similar to an earlier observation, ${ }^{2}$ our results show that the viscosity ratio influences the width of the DC regime. With a decrease in $\lambda$, this zone is limited to $Q$ $\in[1 / 11,1 / 10]$ as compared to $Q \in[1 / 24,1 / 19]$, indicating $\sim 20 \%$ reduction in the flow rate of the continuous phase. In contrast, earlier experimental findings have been reported for $\lambda=0.35$ and 0.07 . $^{2}$ In addition, the width of the DC regime decreases as $\mathrm{Ca}$ increases (Fig. 2). The width of the transition

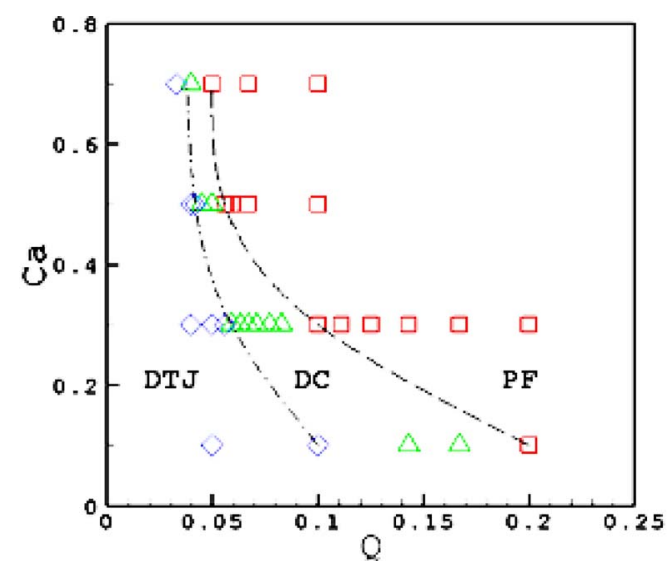

(a)

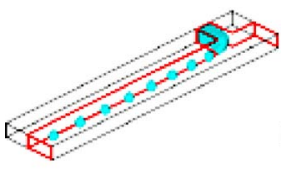

(1) DTJ

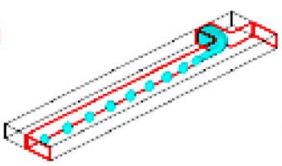

(11) DC

(b)

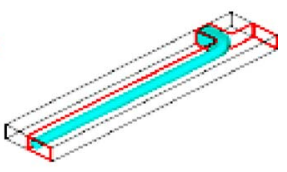

(111) PF
FIG. 2. (Color online) (a) Droplet flow regimes as a function of flow rate ratio $(Q)$ and capillary number $(\mathrm{Ca})$ at viscosity ratio $\lambda=1 / 10$ and (b) representative outcomes for the three regimes at $\mathrm{Ca}=0.3$ and $Q=1 / 20,1 / 14$ and $1 / 7$, respectively. Symbols in (a) indicate $(\square)$ : PF, $(\triangle)$ : DC, and $(\diamond)$ : DTJ. Data at different regimes are shown in different colors only for visual guidelines.

zone DC was found to increase as the Capillary number was lowered until $\mathrm{Ca}=0.1$. Results at an even lower Capillary number, i.e., $\mathrm{Ca}=0.01$, show that the width of the two extreme cases shown by the curves in Fig. 2 corresponds to $Q \in[1 / 2,1]$. $^{16}$

Figure 3 shows normalized droplet volume as a function of capillary number for different flow rate ratios and at fixed $\lambda=1 / 10$. At a fixed $\mathrm{Ca}$, the flow rate ratio was varied by changing the dispersed phase velocity. The droplet volume was normalized using $V_{0}=w_{c}^{2} h .^{17}$ A force analysis is used in the present study adapted from Christopher et al., ${ }^{17}$ where pressure and shear forces are assumed to be the same and the

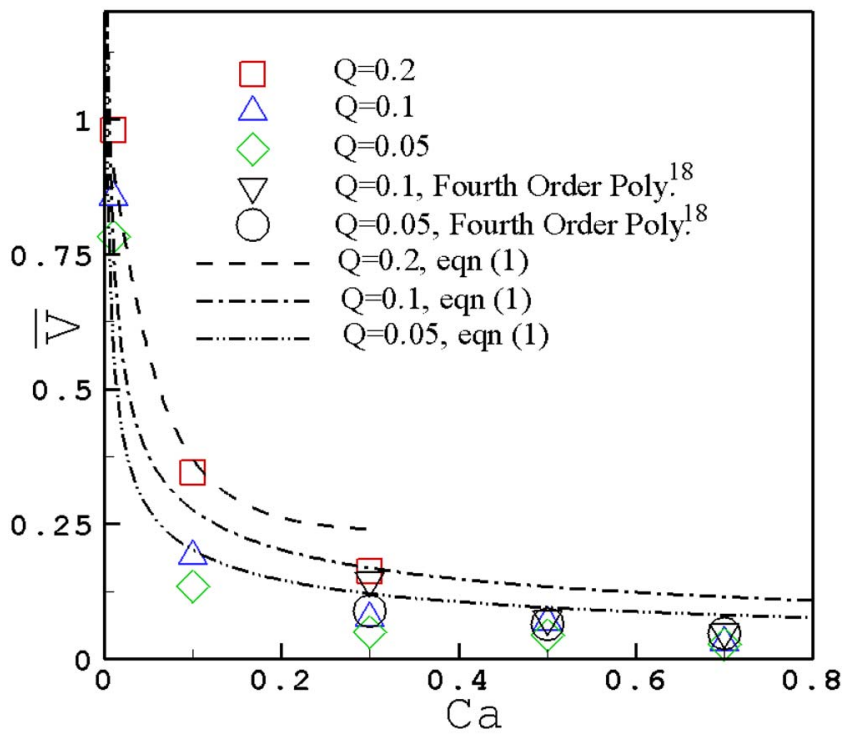

FIG. 3. (Color online) Normalized droplet volume as a function of capillary number for different flow rate ratios. 


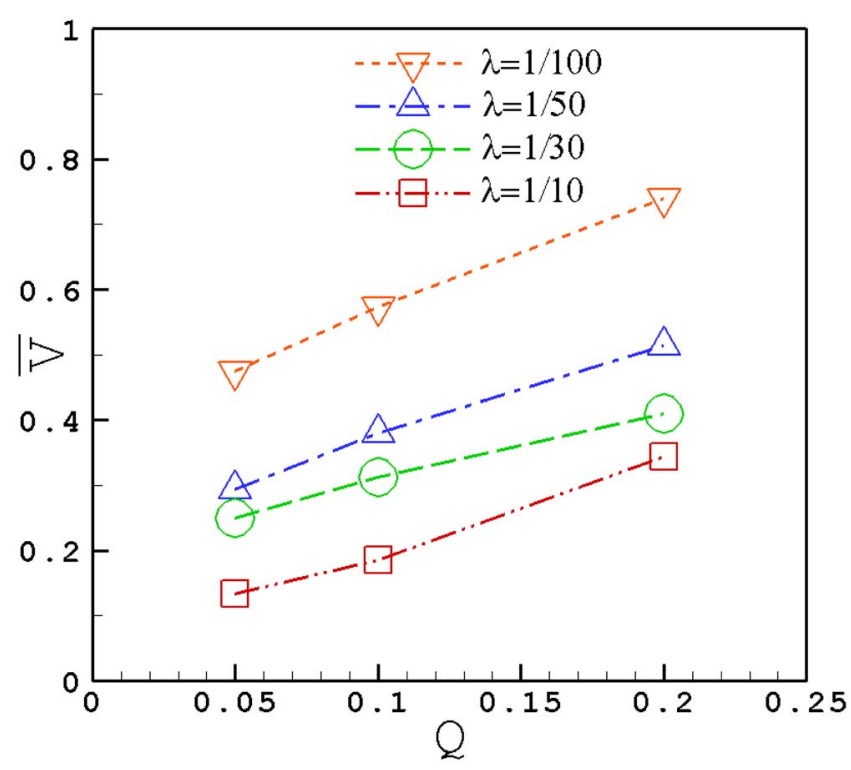

FIG. 4. (Color online) Normalized droplet volume as a function of the flow rate ratio $Q$ for different viscosity ratios.

interfacial force is scaled down by a factor $k,(k<1)$, such that $F_{\sigma}=k \sigma h$. This is to account for the case when the curvature in the depth direction is greater than $2 / h$. On balancing the interfacial, shear and pressure forces, the modified form of the cubic polynomial for $\bar{b}\left(=b / w_{c}\right)$, where $b$ is the length of the drop before it pinches, becomes $k(1-\bar{b})^{3}$ $=\bar{b} \mathrm{Ca}$. Based on this, the volume of the droplet is computed as $^{17}$

$$
\bar{V} \approx \overline{b^{2}}+\Lambda Q
$$

A comparison of our results with the force analysis ${ }^{17}$ and a fourth order polynomial ${ }^{18}$ obtained at pinch off shows reasonable agreement in the order and dependence of droplet volume on $\mathrm{Ca}$.

For the Capillary numbers simulated, the droplet volume decreases as $\mathrm{Ca}$ is increased. The nondimensional volume decreases from 1.0 to nearly 0.2 from $\mathrm{Ca}=0.01$ and 0.1 , beyond which the drop it seems to reach an asymptotic value of nearly 0.1 at Capillary numbers greater than 0.5 . For the same $\mathrm{Ca}$, the droplet volume increases with the flow ratio, a trend seen by others as well. ${ }^{17,18}$ This is due to the fact that the dispersed flow increases as the flow rate ratio is increased, thus enabling a higher volume to be pushed into the expanding droplet.

In order to determine the influence of continuous phase viscosity on droplet volume, simulation results were compared for a range of viscosity ratios at a fixed $\mathrm{Ca}=0.1$ (Fig. 4). Different viscosity ratios of $\lambda=1 / 30,1 / 50$, and $1 / 100$ were chosen by keeping the dispersed phase viscosity the same value as that used for $\lambda=1 / 10$, and the continuous phase viscosity was increased. This results in a lower average velocity of the continuous phase, and hence a lower flow rate $Q_{c}$. For the same geometric variables $w_{c}$ and $w_{d}, \operatorname{Re}_{c}$ decreased by factors of $1 / 9,1 / 25$, and $1 / 100$ corresponding to $\lambda=1 / 30,1 / 50$ and $1 / 100$, respectively, as compared to $\mathrm{Re}_{c}$ for $\lambda=1 / 10$. In the shear dominated regime, the drag force due to viscous effects is dominant as compared to the pressure force due to blockage in the main channel. The drag force (D) is $D \propto C_{D} U_{c}^{2} A$, and can be written in terms of stress as $\tau_{D} \propto C_{D} U_{c}^{2}$. With an increase in the continuous phase viscosity, the increase in the drag coefficient $\left(C_{D}\right)$ does not match the corresponding decrease in $U_{c}^{2}$, resulting in an induced shear drag that is lower as compared to that of a lower continuous phase viscosity fluid. Thus, a smaller drag force would act on the expanding droplet. This will enable the protuberant dispersed phase to expand in the main channel for a longer time before it is pinched off and hence an increase in droplet volume. This analysis agrees with our current results shown in Fig. 4, where the normalized droplet volume is shown to increase as the continuous phase viscosity increases (i.e., $\lambda \downarrow$ ). A power-law curve was fitted to the experimental data of Christopher et al. ${ }^{17}$ for low $\mathrm{Ca}$ as $\bar{V}$ $=Q \mathrm{Ca}^{-0.3} / \bar{\beta}$, where $\bar{\beta}$ is a measure of the capillary breakup frequency. They reported $\bar{\beta}$ to be 1.13 for $\lambda=1 / 50$. In Fig. 4, $\bar{\beta}=1.37$ for the same viscosity ratio, where $\bar{\beta}$ was estimated from the slope of normalized volume versus flow rate ratio. The droplet volume increases when the dispersed flow rate or the continuous flow viscosity increases.

In summary, the transition from droplets formed at the T-junction to parallel flows is strongly dependent on the Capillary number of the continuous phase. As the Capillary number increases, the transition from a droplet flow to parallel flow occurs earlier for a fixed viscosity ratio. For higher viscosities of the continuous phase, the transition zone was found to become narrower, an observation consistent with an earlier experimental study. ${ }^{2}$ The volume of the droplets is a function of the viscosity ratio as well.

The authors would like to acknowledge Dr. Ian Halliday and Dr. Long Wu for helpful discussions.

${ }^{1}$ G. F. Christopher and S. L. Anna, J. Phys. D 40, R319 (2007).

${ }^{2}$ P. Guillot and A. Colin, Phys. Rev. E 72, 066301 (2005).

${ }^{3}$ P. B. Umbanhowar, V. Prasad, and D. A. Weitz, Langmuir 16, 347 (2000).

${ }^{4}$ C. Cramer, P. Fischer, and E. J. Windhab, Chem. Eng. Sci. 59, 3045 (2004)

${ }^{5}$ S. L. Anna, N. Bontoux, and H. A. Stone, Appl. Phys. Lett. 82, 364 (2003).

${ }^{6}$ P. Garstecki, M. J. Fuerstman, and G. M. Whitesides, Phys. Rev. Lett. 94, 234502 (2005).

${ }^{7}$ Y. C. Tan, J. S. Fisher, A. I. Lee, V. Cristini, and A. P. Lee, Lab Chip 4 , 292 (2004).

${ }^{8}$ T. Thorsen, R. W. Roberts, F. H. Arnold, and S. R. Quake, Phys. Rev. Lett. 86, 4163 (2001).

${ }^{9}$ P. Garstecki, M. J. Fuerstman, H. A. Stone, and G. M. Whitesides, Lab Chip 6, 437 (2006).

${ }^{10}$ S. van der Graaf, T. Nisisako, C. G. P. H. Schroën, R. G. M. van der Sman, and R. M. Boom, Langmuir 22, 4144 (2006).

${ }^{11}$ J. D. Tice, A. D. Lyon, and R. F. Ismagilov, Anal. Chim. Acta 507, 73 (2004).

${ }^{12}$ M. De Menech, P. Garstecki, F. Jousse, and H. A. Stone, J. Fluid Mech. 595, 141 (2008).

${ }^{13}$ S. V. Lishchuk, C. M. Care, and I. Halliday, Phys. Rev. E 67, 036701 (2003).

${ }^{14}$ A. K. Gunstensen, D. H. Rothman, S. Zaleski, and G. Zanetti, Phys. Rev. A 43, 4320 (1991).

${ }^{15}$ M. Latva-Kokko and D. H. Rothman, Phys. Rev. E 71, 056702 (2005).

${ }^{16}$ See EPAPS Document No. E-APPLAB-94-084914 for the formation of droplets for $\mathrm{Ca}=0.01$ and $Q=1 / 10$. For more information on EPAPS, see http://www.aip.org/pubservs/epaps.html.

${ }^{17}$ G. F. Christopher, N. N. Noharuddin, J. A. Taylor, and S. L. Anna, Phys. Rev. E 78, 036317 (2008).

${ }^{18}$ J. Husny and J. J. Cooper-White, J. Non-Newtonian Fluid Mech. 137, 121 (2006). 